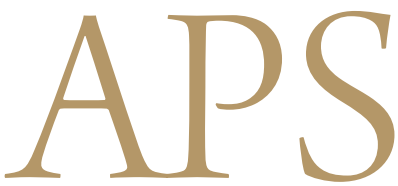

Archives of Plastic Surgery

\title{
Lymphedema Fat Graft: An Ideal Filler for Facial Rejuvenation
}

\author{
Fabio Nicoli ${ }^{1,2,3}$, Ram M. Chilgar ${ }^{2}$, Stamatis Sapountzis ${ }^{2}$, Davide Lazzeri ${ }^{3}$, \\ Matthew Yeo Sze $\mathrm{Wei}^{2}$, Pedro Ciudad ${ }^{2}$, Marzia Nicoli ${ }^{1,3,4}$, Seong Yoon Lim² ${ }^{2}$ Pei-Yu Chen ${ }^{5}$, \\ Joannis Constantinides ${ }^{2,6}$, Hung-Chi Chen ${ }^{2}$
}

${ }^{1}$ Department of Plastic and Reconstructive Surgery, University of Rome "Tor Vergata", Rome, Italy; ${ }^{2}$ Department of Plastic and Reconstructive Surgery, China Medical University Hospital, Taichung, Taiwan; ${ }^{3}$ Plastic Reconstructive and Aesthetic Surgery, Villa Salaria Clinic, Rome, Italy; ${ }^{4}$ Department of Immunology, University of Rome "La Sapienza", Rome, Italy; ${ }^{5}$ Department of Pathology, China Medical University Hospital, Taichung, Taiwan; 'Department of Plastic and Reconstructive Surgery, St. Thomas' Hospital, London, UK

Lymphedema is a chronic disorder characterized by lymph stasis in the subcutaneous tissue. Lymphatic fluid contains several components including hyaluronic acid and has many important properties. Over the past few years, significant research has been performed to identify an ideal tissue to implant as a filler. Because of its unique composition, fat harvested from the lymphedema tissue is an interesting topic for investigation and has significant potential for application as a filler, particularly in facial rejuvenation. Over a 36-month period, we treated and assessed 8 patients with lymphedematous limbs who concurrently underwent facial rejuvenation with lymphedema fat (LF). We conducted a pre- and postoperative satisfaction questionnaire survey and a histological assessment of the harvested LF fat. The overall mean general appearance score at an average of 6 months after the procedure was $7.2 \pm 0.5$, demonstrating great improvement. Patients reported significant improvement in their skin texture with a reading of $8.5 \pm 0.7$ and an improvement in their self-esteem. This study demonstrates that LF as an ideal autologous injectable filler is clinically applicable and easily available in patients with lymphedema. We recommend the further study and clinical use of this tissue as it exhibits important properties and qualities for future applications and research.

Keywords Lymphedema / Adipose tissue / Hyaluronic acid / Rejuvenation / Esthetics
Correspondence: Hung-Chi Chen Department of Plastic and Reconstructive Surgery, China Medical University Hospital, 2 Yuh-Der Road, Taichung 40447, Taiwan Tel: +886422052020

Fax: +886422030777

E-mail: D19722@mail.cmuh.org.tw

This paper was presented at the 24 th International Society of Lymphology Conference on September 16-20, 2013, in Rome, Italy.

No potential conflict of interest relevant to this article was reported.

Received: 17 Mar $2014 \bullet$ Revised: 30 May $2014 \bullet$ Accepted: 14 Jun 2014

plSSN: 2234-6163・elSSN: 2234-6171 • http://dx.doi.org/10.5999/aps.2014.41.5.588・ Arch Plast Surg 2014;41:588-593

\section{INTRODUCTION}

Lower and upper extremity lymphedema can be a debilitating side effect of lymphadenectomy or radiation therapy for gynecological and breast malignancies, respectively. Lymphedema is a build-up of lymph fluid in the fatty tissues just under the skin down to the deep fascia, which deforms and causes heaviness, particularly that of the arms and legs. This results in a profound effect on patients, such as embarrassment over body image and depression, which may lead to social isolation and ultimately compromise the patient's quality of life [1]. Several studies have been conducted to investigate the composition of the interstitial 
fluid from swollen limbs. A biochemical analysis of lymph demonstrated that lymphatic fluid contains several components such as proteins, lipids, glycosaminoglycans, leukocytes, and electrolytes, with different gradients in different pathological states [2-4]. Among glycosaminoglycans, hyaluronic acid (HA) is one of the major components of the interstitium and has a close relationship with the lymphatic system. HA has a significant function in promoting tissue integrity, enabling cell migration during inflammation and wound repair and even in modulating adjacent tissue interaction [3]. Due to its unique features, HA has been extensively used in cosmetic surgery to improve the skin quality and achieve facial rejuvenation. Liu and Zhang [4] have recently reported that the concentration of HA in the interstitial fluid of a lymphedematous limb is significantly higher than that present in the interstitial fluid, serum, and lymph tissue of normal limbs.

Over the last decade, injectable soft tissue fillers have become an indispensable part of facial plastic surgery and many experts are conducting ongoing research to identify an ideal tissue to implant [5]. Because of its unique composition, lymphedema fat (LF) is an interesting tissue for investigation and has significant potential for application, particularly in facial rejuvenation. In this article, we present the use of an LF graft for the improvement of skin quality and facial rejuvenation in patients undergoing lymphedema surgery. We assessed the level of patient satisfaction and reviewed the long-term results, particularly regarding volume contour changes and textural improvements of the skin. Furthermore, we evaluated the histological differences between $L F$ and normal fat.

\section{IDEA}

Over a 36-month period, a total of 8 patients had undergone facial rejuvenation with an LF graft at China Medical University
Hospital in Taichung, Taiwan. Patients with an advanced stage of lymphedema (more than Hung-Chi Chen stage III B) undergoing a debulking surgical treatment were eligible for this procedure when an improvement of facial aging changes was also desired [6]. The patients' average age was 54 years (range, 45-62 years). All patients were female. Each patient was fully informed and provided written consent regarding the aim of the study and treatment and the possible complications. All patients underwent a single LF grafting procedure. Patient data are presented in Table 1. As part of our clinical routine, data and clinical photographs were collected. The outcome analysis consisted of a patient satisfaction survey and data collection regarding postoperative complications and the amount of time to full recovery. A custom-made questionnaire was used, consisting of 5 questions to assess patient satisfaction before and 6 months after surgery. We recorded each answer on a subjective analysis scale of $0-10$, where 0 was minimum and 10 was maximum. We repeated the satisfaction survey 6 months after surgery. The questionnaire included the following items:

1) How do you rate the general appearance of your face?

2) How do you rate your skin texture including the color, brightness, and firmness of skin?

3) What do you think of the general appearance of your facial wrinkles and rhytides?

4) How do you rate your self-esteem in terms of your overall confidence level and comfort with yourself?

5) Are you comfortable socializing with friends and relatives?

A statistical analysis was performed in terms of the mean and standard deviation, and the data were extracted from the subjective assessment of patients, who responded before treatment and 6 months postoperatively to the abovementioned questionnaire. Student's t-test was used for a comparison of the preoperative and postoperative scores; $\mathrm{P}<0.05$ was considered to be statistically significant.

Table 1. Patient summary

\begin{tabular}{|c|c|c|c|c|c|c|c|}
\hline No. & Age/Gender & Lymphedema region/Cause & HCC staging & Surgical procedure & LFG quantity (mL) & Complications & Follow-up (mo) \\
\hline 1 & 45/Female & Arm/Breast cancer & III B & $\begin{array}{l}\text { LN transfer+ } \\
\text { Liposuction }\end{array}$ & 30 & Ecchymosis & 6 \\
\hline 2 & 49/Female & Leg/Endometrial cancer & IV A & LN transfer+charles procedure & 35 & None & 36 \\
\hline 3 & 52/Female & Arm/Breast cancer & III B & $\begin{array}{l}\text { LN transfer+ } \\
\text { Liposuction- }\end{array}$ & 25 & None & 12 \\
\hline 4 & 53/Female & Leg/SCC vulva & III B & LN transfer+RRPP+liposuction & 30 & None & 12 \\
\hline 5 & 54/Female & Leg/Foot melanoma & III B & RRPP+Liposuction & 35 & None & 6 \\
\hline 6 & 56/Female & Leg/Cervical cancer & IV A & LN transfer+charles procedure & 40 & None & 36 \\
\hline 7 & 58/Female & Arm/Breast cancer & III B & $\begin{array}{l}\text { LN transfer+ } \\
\text { Liposuction+excision }\end{array}$ & 35 & None & 24 \\
\hline 8 & 62/Female & Leg/Cervical cancer & III B & RRPP+liposuction & 40 & Edema & 12 \\
\hline
\end{tabular}

HCC, Hung-Chi Chen; LFG, lymphedema fat graft; LN, lymph node; SCC, squamous cell carcinoma; RRPP, radical reduction with preservation of perforators. 


\section{Fig. 1. Case 1: Preoperative, intraoperative, and postoperative photographs}

(A) Lymphedema fat graft rejuvenation case: preoperative photograph. (B) Intraoperative photograph of lymphedema fat harvesting. (C) Clinical case at postoperative year 2 .
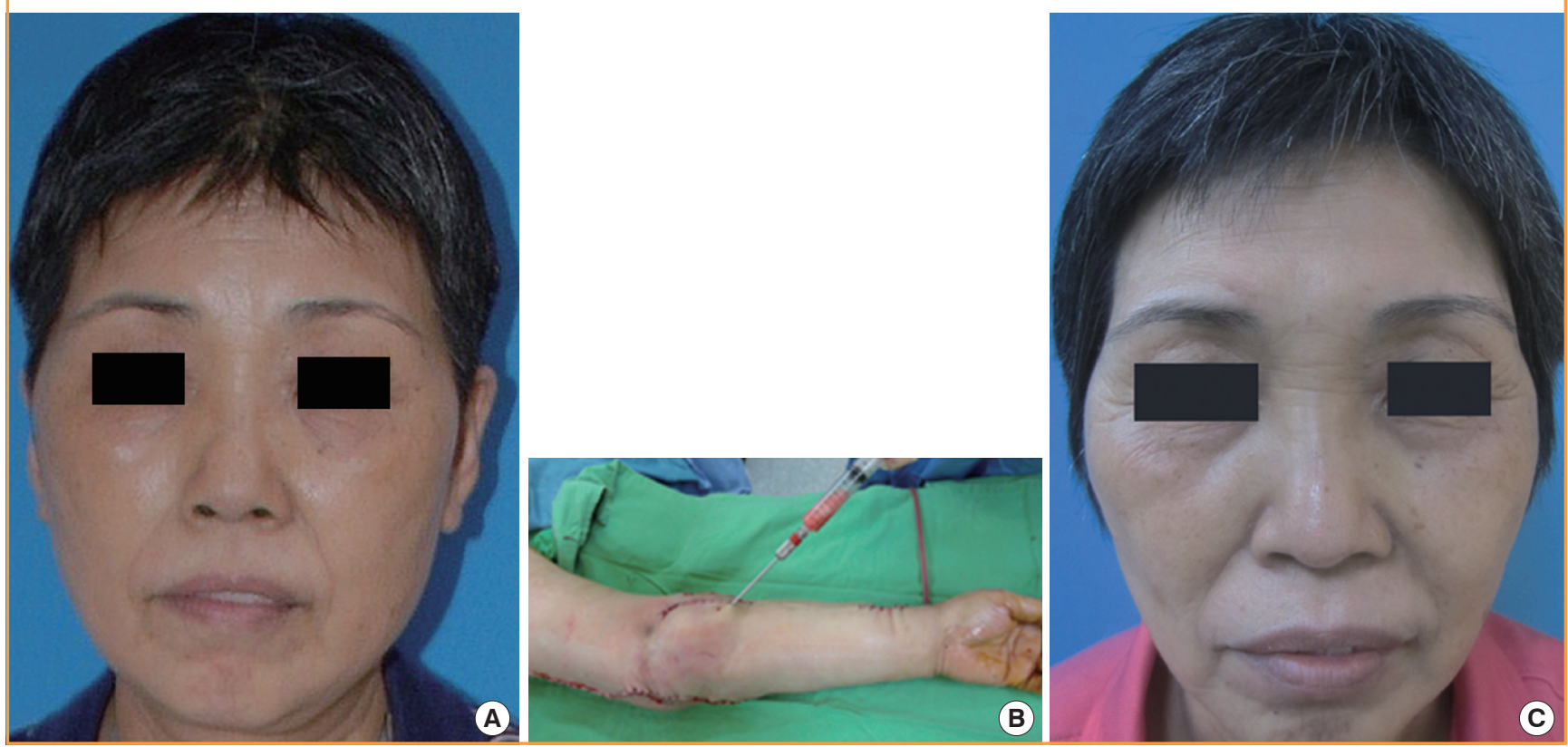

Table 2. Subjective assessment

\begin{tabular}{lcccc}
\hline Assessment parameters & Preoperative $^{\text {a) }}\left(\right.$ mean $_{ \pm}$SD) & Range & Postoperative $^{\text {a) }}\left(\right.$ mean $_{ \pm}$SD) & Range \\
\hline Appearance of face & $4 \pm 0.7$ & $3-5$ & $7.2 \pm 0.5$ & $7-8$ \\
Skin texture & $4.2 \pm 0.5$ & $4-5$ & $8.5 \pm 0.7$ & $8-10$ \\
Wrinkles and rhytides & $5 \pm 1$ & $4-6$ & $7.4 \pm 0.5$ & $7-8$ \\
Self-esteem & $3.5 \pm 0.5$ & $3-4$ & $7.2 \pm 0.5$ & $7 \pm 0.5$ \\
Socialization & $5 \pm 0.5$ & $4-6$ & $7.46 \pm 0.59$ & \\
P $<0.01$ & $4.34 \pm 0.65$ & & & \\
\hline SD, standard deviation. & & & & \\
a) Score: minimum, 0; maximum, 10. & & & & \\
\hline
\end{tabular}

\section{Lymphedema fat harvesting}

All the procedures were performed under general anesthesia. The patient donor areas were the lymphedematous limbs (arm or leg). LF was harvested using a $10-\mathrm{mL}$ syringe for hand-assisted aspiration and a standard cannula (Fig. 1A, B). After sedimentation of the aspirate, we discarded the top layer of the sediment containing a tumescent fluid, blood, and serum cell fragments, while the LF tissue remaining in HA was transferred to 1-mL syringes for the injections [4]. Washing and centrifugation were not performed. A specimen of LF was sent to the laboratory for a histological evaluation. Another specimen of fat was harvested from the abdomen with a similar modality and sent to the laboratory for a comparative analysis of the tissues.

\section{Lymphedema fat grafting}

We injected the LF in multiple planes by using fanning and cross-hatching techniques in the pre-marked areas. Marking was performed preoperatively in a sitting position. Injection of tissue was typically performed with a deposition of small amounts of LF via multiple passes deeper into the rhytides of the glabella and forehead. For superficial lines, particularly in the crow's feet region, prejowl sulcus, nasolabial folds, and marionette lines and to correct the irregular contours of the chin and the nose, we usually inject a total of $25-40 \mathrm{~mL}$ of LF. A total of 8 patients underwent facial rejuvenation using the LF graft technique over a period of 36 months. The facial area treated included the forehead, glabella, periorbital areas, malar area, buccal area, lips, nasolabial folds, and mental area. There were no major procedurerelated complications at the donor or recipient sites except ecchymosis in the malar region in one patient and persistent edema in another patient, which was managed with conservative treatment. The patients' questionnaire responses were collected and are presented in Table 2 . The overall mean general appearance score at an average of 6 months after the procedure was 
Fig. 2. Case 2: preoperative and postoperative photographs

(A) Lymphedema fat graft rejuvenation case: preoperative photograph. (B) Photograph at follow-up at postoperative year 1 .
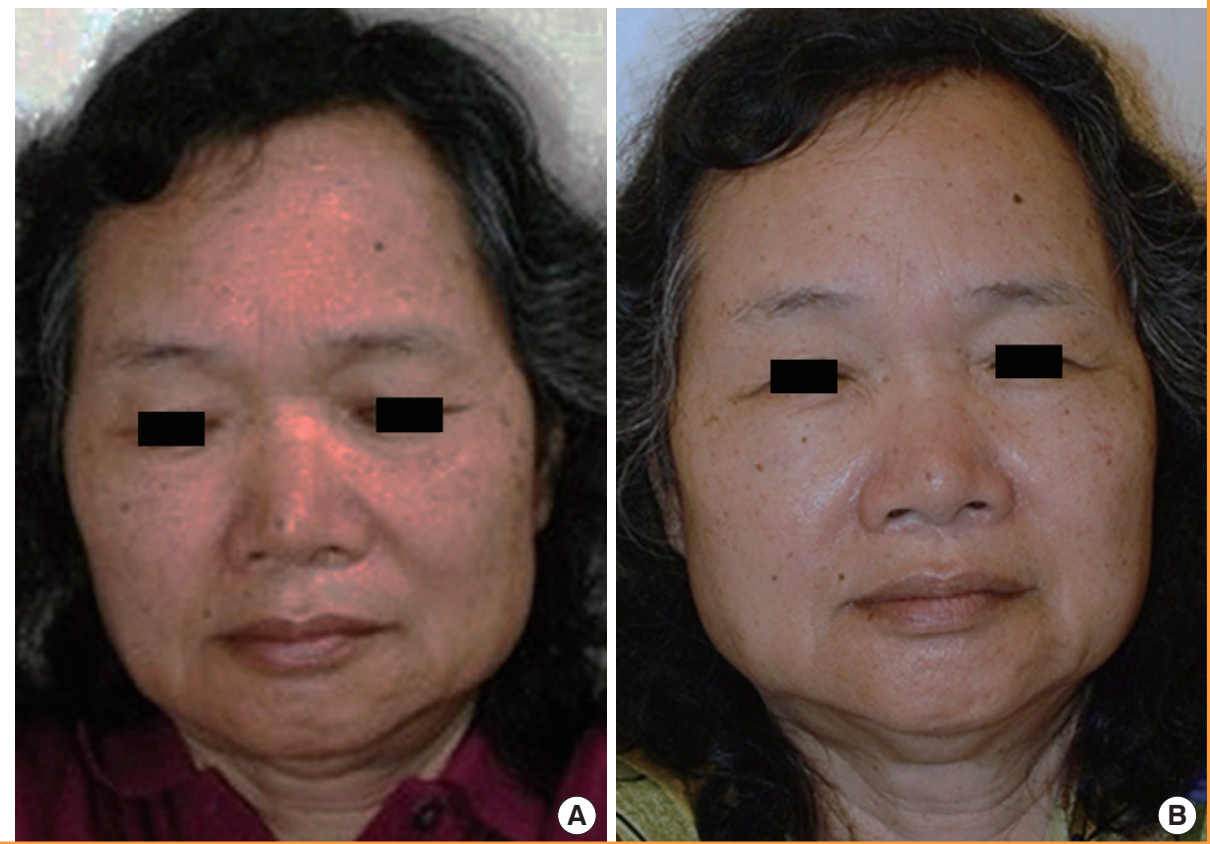

\section{Fig. 3. Pathologic comparison between fat and lymphedema fat}

(A) Histological analysis of abdominal fat: Masson's Trichrome stain on $\times 40$ magnification. (B) Histological analysis of lymphedema fat: Masson's Trichrome stain on $\times 40$ magnification.

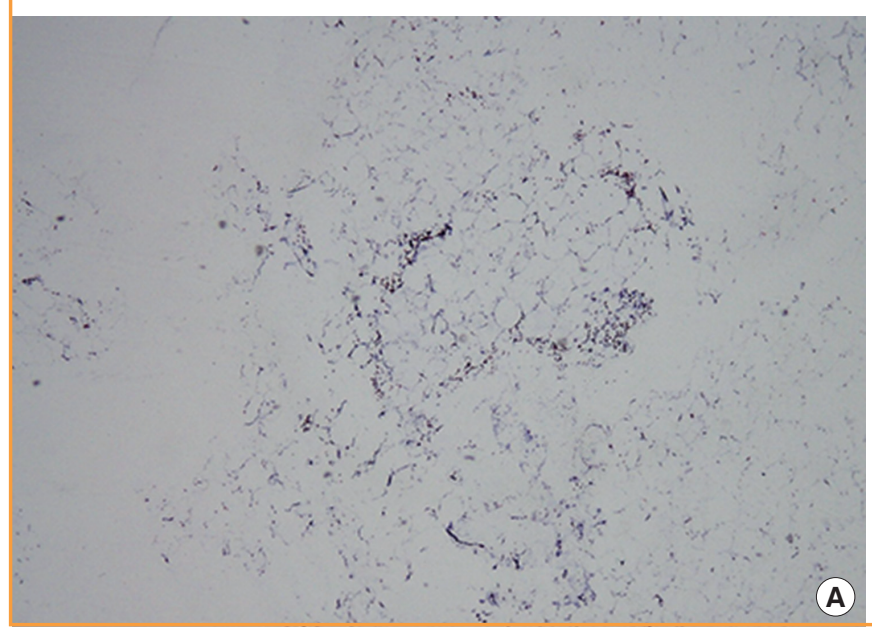

$7.2 \pm 0.5$. The patients reported a great improvement in the skin texture with a reading of $8.5 \pm 0.7$ on the subjective analysis scale. With respect to the psychological parameters, the patients had a significant improvement in their self-esteem with an average of $7.2 \pm 0.5$. The overall improvement in other parameters made socializing more comfortable than before the treatment (mean, $7 \pm 0.5$ ). Student's t-test was applied to the preoperative and postoperative scores, and no significant difference was found $(\mathrm{P}<0.01)$. Clinical examples are shown in Fig. 1A, C, and 2. A comparative histological analysis was performed with Masson's Trichrome staining on the fat specimen harvested

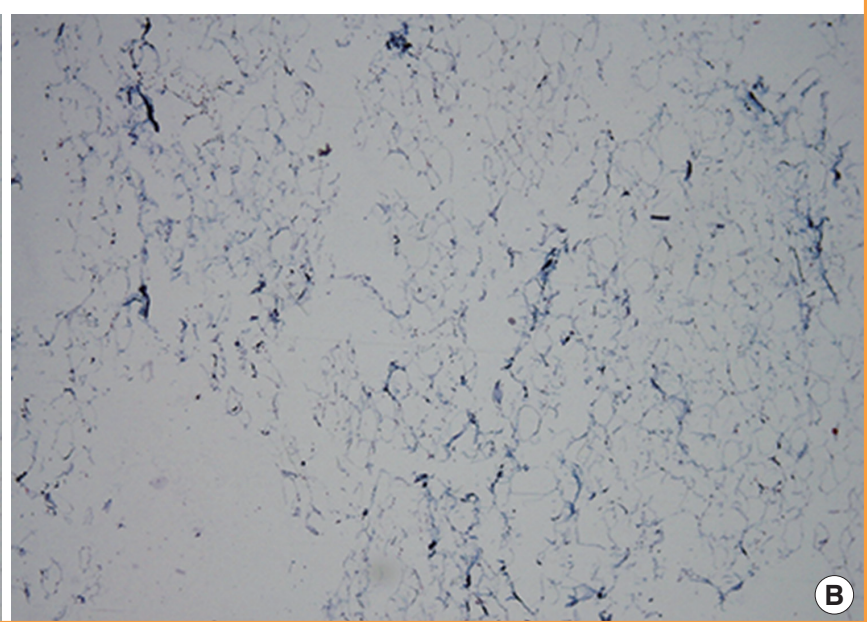

from the abdomen (Fig. 3A) and on the LF harvested from the pathological limb (Fig. 3B). The area stained blue with the Masson's trichrome stain on $40 \times$ magnification showed great numbers of adipose cells and a large number of collagen fibers on the LF specimen (Fig. 3B).

\section{DISCUSSION}

Many plastic surgeons work for years trying to prevent the aging process of their patients and improve the age-related appearance of the face by using various injectable agents including synthetic 
and autologous agents and to search for an ideal soft tissue filler. A review of fillers in 2007 described an ideal filler material as biocompatible, non-antigenic, non-toxic, easy to use, long-lasting (yet not permanent), inexpensive, and reversible. Its safety profile should be high, and a predictable result should be achievable [7]. Facial autologous fat transfer has become one of the most popular methods for facial rejuvenation because fat possesses many qualities of an ideal filler, including its lack of immunogenicity, relatively low cost, abundant donor-tissue availability, and ease of harvesting [8]. However, the notable problems that have limited its widespread acceptance are the unpredictable absorption rates causing contour irregularities, which require multiple repetitions of the procedure, potential dissipation of the transplanted fat, and the risk of lumps and bulges along thin skin areas such as the periorbital area [8,9]. Some studies report $20 \%$ to $50 \%$ postgrafting resorption commonly observed with autologous fat grafting [9]. Several substances such as growth factors, beta blockers, insulin, and hyperbaric oxygen, and even the combined use of adipose-derived stem cells and platelet-rich plasma synergistically supplement fat grafts, increasing revascularization and fat graft survival, improving cosmetic facial contouring, and decreasing complications [10]. Recently, Levi et al. [11] demonstrated that lymphedema-associated adipose-derived stem cells have a much higher adipogenic gene expression and enhanced ability to undergo adipogenic differentiation compared to control adipose-derived stem cells. Alghoul et al. [12] demonstrated in an experimental model that the mixing of HA with the fat graft can potentially improve early fat graft survival and thus, enhance vascularity and prolong fat volume maintenance. This demonstrates the ability of LF to last for a long period of time, and patients, even after many months of the procedure, are satisfied with the results. HA is an important component of the extracellular matrix, which is synthesized in the interstitial tissue, transported in the lymph, and catabolized mainly in lymph nodes and the liver. HA acts by binding water molecules, resulting in increased skin hydration and turgor. Its hydrophilic properties help with volume maintenance and tissue viscoelasticity. HA, since 1996 when it was launched in the European market, was utilized as a filler agent for improving wrinkles and scars, or increasing the tissue volume, and has since become the "gold standard" for fillers [13].

Recently, in a clinical investigation, Liu et al. [2-4] found that the hyaluronan content in the interstitial fluid of the lymphedematous limb is significantly higher than that of a normal limb. These data further validate the use of an LF graft in vitro by using an experimental model [14]. We performed a histological analysis of the harvested LF (as shown in Fig. 3B), which in accordance to the literature and in comparison to the normal fat harvested from the abdomen (Fig. 3A), demonstrates the presence of a large quantity of collagen, elastin, and adipocytes. The LF tissue contains high levels of HA and is rich in precious antiaging molecules. Furthermore, recent experimental studies have shown that lymphatic fluid stasis results in a subcutaneous fat deposition and that this effect is associated with increased adipocyte number, lipid accumulation, and fibrosis [15]. Therefore, lymphedematous patients have available a large amount of fat to harvest and are rich in HA and stem cells with a high adipogenic potential that can be used for different clinical purposes.

This study demonstrated a clinical application of LF as an ideal autologous injectable filler easily available in patients with lymphedema. This method could be rightly offered to lymphedema patients simultaneously with other surgical or non-surgical interventions in order to boost their quality of life. Furthermore, we recommend the further study and investigation of this tissue having important properties and qualities for future clinical applications and research.

\section{REFERENCES}

1. Shih YC, Xu Y, Cormier JN, et al. Incidence, treatment costs, and complications of lymphedema after breast cancer among women of working age: a 2-year follow-up study. J Clin Oncol 2009;27:2007-14.

2. Liu N. Metabolism of macromolecules in tissue. Lymphat Res Biol 2003;1:67-70.

3. Liu NF. Trafficking of hyaluronan in the interstitium and its possible implications. Lymphology 2004;37:6-14.

4. Liu NF, Zhang LR. Changes of tissue fluid hyaluronan (hyaluronic acid) in peripheral lymphedema. Lymphology 1998; 31:173-9.

5. Coleman SR. Structural fat grafts: the ideal filler? Clin Plast Surg 2001;28:111-9.

6. Salgado CJ, Mardini S, Spanio S, et al. Radical reduction of lymphedema with preservation of perforators. Ann Plast Surg 2007;59:173-9.

7. Rohrich RJ, Ghavami A, Crosby MA. The role of hyaluronic acid fillers (Restylane) in facial cosmetic surgery: review and technical considerations. Plast Reconstr Surg 2007;120: 41S-54S.

8. Lam SM, Glasgold RA, Glasgold MJ. Limitations, complications, and long-term sequelae of fat transfer. Facial Plast Surg Clin North Am 2008;16:391-9.

9. Pinski KS, Roenigk HH Jr. Autologous fat transplantation. Long-term follow-up. J Dermatol Surg Oncol 1992;18:17984.

10. Keyhan SO, Hemmat S, Badri AA, et al. Use of platelet-rich 
fibrin and platelet-rich plasma in combination with fat graft: which is more effective during facial lipostructure? J Oral Maxillofac Surg 2013;71:610-21.

11. Levi B, Glotzbach JP, Sorkin M, et al. Molecular analysis and differentiation capacity of adipose-derived stem cells from lymphedema tissue. Plast Reconstr Surg 2013;132:580-9.

12. Alghoul M, Mendiola A, Seth R, et al. The effect of hyaluronan hydrogel on fat graft survival. Aesthet Surg J 2012;32: 622-33.

13. Andre P. Hyaluronic acid and its use as a "rejuvenation" agent in cosmetic dermatology. Semin Cutan Med Surg 2004;23:218-22.

14. Liu N, Shao L, Xu X, et al. Hyaluronan metabolism in rat tail skin following blockage of the lymphatic circulation. Lymphology 2002;35:15-22.

15. Aschen S, Zampell JC, Elhadad S, et al. Regulation of adipogenesis by lymphatic fluid stasis: part II. Expression of adipose differentiation genes. Plast Reconstr Surg 2012;129: 838-47. 